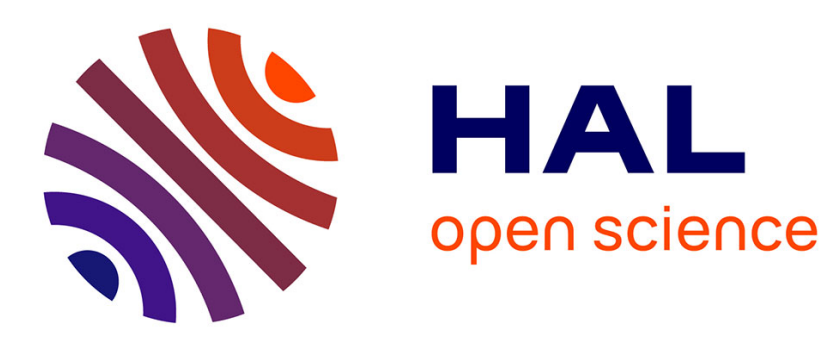

\title{
Stationary Solution to the Fluid Queue Fed by an $\mathrm{M} / \mathrm{M} / 1$ Queue
}

Nelly Barbot, Bruno Sericola

\section{To cite this version:}

Nelly Barbot, Bruno Sericola. Stationary Solution to the Fluid Queue Fed by an M/M/1 Queue. [Research Report] RR-4281, INRIA. 2001. inria-00072306

\section{HAL Id: inria-00072306 https://hal.inria.fr/inria-00072306}

Submitted on 23 May 2006

HAL is a multi-disciplinary open access archive for the deposit and dissemination of scientific research documents, whether they are published or not. The documents may come from teaching and research institutions in France or abroad, or from public or private research centers.
L'archive ouverte pluridisciplinaire HAL, est destinée au dépôt et à la diffusion de documents scientifiques de niveau recherche, publiés ou non, émanant des établissements d'enseignement et de recherche français ou étrangers, des laboratoires publics ou privés. 


\section{Stationary Solution to the Fluid Queue Fed by an M/M/1 Queue}

Nelly Barbot, Bruno Sericola

\section{$\mathbf{N}^{\circ} 4281$}

Octobre 2001

THÈME 1 



\title{
Stationary Solution to the Fluid Queue Fed by an $\mathrm{M} / \mathrm{M} / \mathbf{1}$ Queue
}

\author{
Nelly Barbot*, Bruno Sericola* \\ Thème 1 - Réseaux et systèmes \\ Projet Armor
}

Rapport de recherche $\mathrm{n}^{\circ} 4281$ - Octobre 2001 - 16 pages

\begin{abstract}
We consider an infinite capacity buffer receiving fluid at a rate depending on the state of an $\mathrm{M} / \mathrm{M} / 1$ queue. We obtain a new analytic expression for the joint stationary distribution of the buffer level and the state of the $M / M / 1$ queue. This expression is obtained by the use of generating functions which are explicitly inverted. The case of a finite capacity fluid queue is also considered.
\end{abstract}

Key-words: Fluid queue; M/M/1 queue; generating functions

(Résumé : tsvp)

* $\{$ Nelly.Barbot $\}\{$ Bruno.Sericola $\} @$ irisa.fr

Unité de recherche INRIA Rennes

IRISA, Campus universitaire de Beaulieu, 35042 RENNES Cedex (France)

Téléphone : 0299847100 - International : +33299847100

Télécopie : 0299847171 - International : +33299847171 


\section{Solution stationnaire d'une file d'attente fluide alimentée par une file $\mathrm{M} / \mathrm{M} / \mathbf{1}$}

Résumé : On considère un réservoir de capacité infinie recevant du fluide selon un taux dépendant de l'état d'une file $\mathrm{M} / \mathrm{M} / 1$. Nous obtenons une nouvelle expression analytique de la distribution stationnaire jointe du niveau du réservoir fluide et de l'état de la file M/M/1. Cette expression est obtenue par le biais de fonctions génératrices qui sont explicitement inversées. Le cas de la file d'attente fluide de capacité finie est également considéré.

Mots-clé : File d'attente fluide, file $\mathrm{M} / \mathrm{M} / 1$, fonctions génératrices. 


\section{Introduction}

Markov modulated fluid flow models have turned out to be very useful to analyze performance issues in telecommunication systems. These models are composed of a buffer and a continuous time Markov chain that controls the input and service rates of the fluid in the buffer. In most studies dealing with the analysis of such fluid queues, the state space of the background Markov chain is supposed to be finite, see for instance [4, 8] and the references therein. We consider here an infinite capacity fluid queue where the input rate is a function of the state of the server in an $\mathrm{M} / \mathrm{M} / 1$ queue and where the service rate is constant. As suggested in [10], this model might represent a Poisson stream of packet arrivals, where the packet length is exponentially distributed. This stream is buffered in a queue and served with a constant rate. The output process of this $\mathrm{M} / \mathrm{M} / 1$ queue forms the input process of the fluid queue.

The stationary behavior of that fluid queue has been analyzed in several papers. Although approaches are different, the fluid level distribution is generally obtained as an integral expression. First, in [10], Virtamo and Norros solve the well-known infinite differential system by studying the continuous spectrum of a key matrix. Secondly, Adan and Resing [1] consider the background process as an alternating renewal process, corresponding to the successive idle and busy periods of the $\mathrm{M} / \mathrm{M} / 1$ queue. By renewal theory arguments, the fluid level distribution is given in terms of integral of Bessel functions. They also obtain the expression of Virtamo and Norros via an integral representation of Bessel functions.

More general input processes are applied to that fluid model. Van Doorn and Scheinhart study in [9] a fluid queue fed by an infinite state birth-death process. They solve the infinite differential system by the use of orthogonal polynomials with respect to a signed measure which is explicitly given in the case of the $\mathrm{M} / \mathrm{M} / 1$ queue and leads to the same integral expression obtained in [10] and [1]. In [7], the authors consider a fluid queue driven by a general Markovian queue with the hypothesis that only one state has a negative drift. By using the differential system, the fluid level distribution is obtained in terms of a series and coefficients computed by means of recurrence relations. Moreover, this study is extended to the finite buffer case in [6]. Finally, the transient distribution of that fluid queue and the convergence to the stationary distribution has been analyzed in [2].

In this paper, we obtain a new analytic expression for the joint stationary distribution of the buffer level and the state of the $\mathrm{M} / \mathrm{M} / 1$ queue. This expression is obtained by writing the solution in terms of a matrix exponential and then by using generating functions that are explicitly inverted. In the following section, we present the model and a matrix analytic

$\mathrm{RR} \mathrm{n}^{\circ} 4281$ 
expression of the solution in terms of a series. In Section 3, we derive the generating function associated with the successive powers of a key matrix used in the solution. Next, in Section 4 , by using the method developed in [3], this generating function is explicitly inverted and we obtain a very simple expression of the joint distribution. In Section 5, we use these results and those of [6] to obtain a similar expression for the case of a finite capacity fluid queue.

\section{Formulation of the model}

We consider a fluid queue with an infinite buffer for which the service rate is a constant $c>0$, and the input rate is governed by an $\mathrm{M} / \mathrm{M} / 1$ queue with arrival rate $\lambda$ and service rate $\mu$. During the busy periods of the $\mathrm{M} / \mathrm{M} / 1$ queue, the input rate in the fluid queue is positive, denoted by $r$, while during the idle periods no fluid enters the queue. We suppose that $r>c$ to avoid the trivial case where the queue remains always empty.

We denote by $\left\{X_{t}, t \geq 0\right\}$ the continuous time Markov chain counting the number of customers in the $\mathrm{M} / \mathrm{M} / 1$ queue. Its infinitesimal generator is denoted by $A$. The non-zero entries of the matrix $A$ are $A_{0,0}=-\lambda, A_{0,1}=\lambda$ and for $j \geq 1$,

$$
A_{j, j-1}=\mu, A_{j, j}=-(\lambda+\mu), A_{j, j+1}=\lambda .
$$

The drifts of that fluid queue represent the difference between the input and service rates. Let $d_{j}$ be the drift when the $\mathrm{M} / \mathrm{M} / 1$ queue is in state $j$. We thus have $d_{0}=-c$ and $d_{j}=r-c$, for every $j \geq 1$. We denote by $D$ the diagonal matrix containing these drifts.

Since we are concerned by the stationary behavior of that fluid queue, we suppose that the stability condition is satisfied, that is

$$
\rho=\frac{\lambda r}{\mu c}<1
$$

where $\rho$ is the traffic intensity. We denote respectively by $X$ and $Q$ the stationary state of the Markov chain $\left\{X_{t}, t \geq 0\right\}$ and the stationary amount of fluid in the buffer.

Let $F_{j}(x)=\operatorname{Pr}\{X=j, Q \leq x\}$. It is easy to see that for $j \geq 1$, we have $F_{j}(0)=0$ and it has been shown in [7] that $F_{0}(0)=1-\rho$. It is well-known, see for instance [10], that the functions $F_{j}$ satisfy, for $x>0$, the following system of differential equations

$$
\begin{aligned}
-c F_{0}^{\prime}(x) & =-\lambda F_{0}(x)+\mu F_{1}(x) \\
(r-c) F_{j}^{\prime}(x) & =\lambda F_{j-1}(x)-(\lambda+\mu) F_{j}(x)+\mu F_{j+1}(x) \text { for } j \geq 1,
\end{aligned}
$$


where $F_{j}^{\prime}(x)$ denotes the derivative of $F_{j}(x)$ with respect to $x$. Let $F(x)$ be the infinite row vector containing the $F_{j}(x)$. This system can also be written as $F^{\prime}(x)=F(x) A D^{-1}$ and its solution is given by

$$
F(x)=F(0) \exp \left(A D^{-1} x\right) .
$$

Let $I$ be the identity matrix. Using a method similar to the uniformization technique, we introduce the matrix $T$ defined by $T=I+A D^{-1} / \theta$, where $\theta=(\lambda+\mu) /(r-c)$. We then have, from (2), for every $j \geq 0$,

$$
\begin{aligned}
F_{j}(x) & =(1-\rho)\left(\exp \left(A D^{-1} x\right)\right)_{0, j} \\
& =(1-\rho) \exp (-\theta x)(\exp (\theta T x))_{0, j} \\
& =(1-\rho) \sum_{n=0}^{\infty} \exp (-\theta x) \frac{(\theta x)^{n}}{n !} T_{0, j}^{n}
\end{aligned}
$$

where $T_{0, j}^{n}$ denotes the $(0, j)$ entry of matrix $T^{n}$.

In what follows, we focus on the calculation of $T_{0, j}^{n}$ using generating functions.

\section{Generating functions}

In this section, we first we recall the definition and properties of the generating functions which are then used in a second subsection to obtain the generating function associated with the matrix $T$.

\subsection{Definition and properties}

Let us consider the complex matrices $M$ indexed on $\mathbb{N} \times \mathbb{N}$. We define

$$
\nu(M)=\sup _{i \in \mathbb{N}} \sum_{j=0}^{\infty}\left|M_{i j}\right|
$$

and denote by $\mathcal{M}$ the set of infinite complex matrices $M$ such that $\nu(M)$ is finite. $\nu$ is a norm on $\mathcal{M}$ and $(\mathcal{M}, \nu)$ is a Banach algebra. With each $M \in \mathcal{M}$, we associate the complex function $\Phi_{M}$, called potential kernel of $M$ or generating function, defined by

$$
\Phi_{M}(z)=\sum_{k=0}^{\infty} M^{k} z^{k}
$$

$\mathrm{RR} \mathrm{n}^{\circ} 4281$ 
for all $z$ such that $|z|<1 / \nu(M)$. Note that for $M \in \mathcal{M}$ and $z$ such that $|z|<1 / \nu(M)$, we have $\Phi_{M}(z) \in \mathcal{M}$ since $\nu\left(\Phi_{M}(z)\right) \leq 1 /(1-|z| \nu(M))<+\infty$.

The following lemma is a classical straightforward result, so we give it without proof.

Lemma 1 For every matrix $H, H \Phi_{M}$ is the only solution to the matrix equation

$$
X(z)=H+z X(z) M
$$

for all z such that $|z|<1 / \nu(M)$.

We shall also need the following result which will be used along with Lemma 1.

Lemma 2 For every $M$ and $N$ in $\mathcal{M}$, we have

$$
\Phi_{M+N}(z)=\Phi_{M}(z)+z \Phi_{M+N}(z) N \Phi_{M}(z)
$$

for all $z$ such that $|z|<\min \{1 / \nu(M), 1 / \nu(M+N)\}$.

Proof. See [3].

Let us now introduce some notation. We define the infinite matrices $V, W, R, S$ and $P$ as

$$
V_{i, j}=I_{i+1, j} ; W_{i, j}=I_{i, j+1} ; R_{i, j}=I_{i, 0} I_{0, j} ; S_{i, j}=I_{i, 1} I_{0, j}
$$

for $i$ and $j \in \mathbb{N}$ and

$$
P=I+\frac{A}{\lambda+\mu}
$$

The matrix $P$ is referred to as the transition probability matrix of the uniformized Markov chain associated with the $\mathrm{M} / \mathrm{M} / 1$ queue. If $p$ and $q$ are defined by

$$
p=\frac{\lambda}{\lambda+\mu} \text { and } q=\frac{\mu}{\lambda+\mu}
$$

then the non-zero entries of $P$ are

$$
P_{0,0}=q, P_{0,1}=p \text { and for } i \geq 1, P_{i, i-1}=q, P_{i, i+1}=p .
$$

The stability condition $\rho<1$ and the fact that $r>c$ implies that $\lambda<\mu$ and so $p<q$. 
Lemma 3 We have $T=P+U$ where $U=(p R-q S) r / c$.

Proof. From the definition of $T$, it suffices to write $A$ and $D^{-1}$ as

$$
\begin{aligned}
A & =(\lambda+\mu)(P-I) \\
D^{-1} & =\frac{1}{r-c}\left(I-\frac{r}{c} R\right)
\end{aligned}
$$

and to use the fact that $P R=q R+q S$.

It is easy to check that

$$
\nu(T)=\max \{1+p r / c, p+q(r-c) / c\}>1 .
$$

Using Lemma 2, we obtain

$$
\Phi_{T}(z)=\Phi_{P}(z)+z \Phi_{T}(z) U \Phi_{P}(z)
$$

for all $z$ such that $|z|<1 / \nu(T)$, since $\nu(P)=1$. We define the matrix $L(z)$ as

$$
L(z)=U \Phi_{P}(z) .
$$

For $|z|<1$, we have

$$
\nu(L(z))=\nu\left(U \Phi_{P}(z)\right) \leq \frac{\nu(U)}{1-|z|}=\frac{r q}{c(1-|z|)}
$$

so for $|z|<c /(q r+c)$ we have $|z|<1 / \nu(L(z))$ and $L(z) \in \mathcal{M}$. Thus, we may apply Lemma 1 to Relation (4) with $X(z)=\Phi_{T}(z), H=\Phi_{P}(z), M=L(z)$ and we get

$$
\Phi_{T}(z)=\Phi_{P}(z) \Phi_{L(z)}(z)
$$

for $|z|<c /(q r+c)$.

\subsection{Calculation of $\Phi_{T}$}

In this section, we derive a simple expression of the potential kernel $\Phi_{T}$ given in Relation (5). We first recall the expression of $\Phi_{P}$ that has been obtained in [3]. 
The Catalan numbers $c_{n}$ are defined for every $n \in \mathbb{N}$ by

$$
c_{n}=\left(\begin{array}{c}
2 n \\
n
\end{array}\right) \frac{1}{n+1} .
$$

The generating function associated with the sequence of these numbers

$$
C(z)=\sum_{n=0}^{\infty} c_{n} z^{n}
$$

converges for all $z$ such that $|z| \leq 1 / 4$ and can be written as

$$
C(z)=\frac{1-\sqrt{1-4 z}}{2 z}
$$

Lemma 4 Let $|z|<1$ and $\eta(z)=C\left(p q z^{2}\right)$. Let $X(z)$ and $Y(z)$ be the infinite matrices defined by

$$
X_{i, j}(z)=(q z \eta(z))^{i}(p z \eta(z))^{j}
$$

and

$$
Y(z)=\sum_{k=0}^{\infty} W^{k} X(z) V^{k}
$$

For all z such that $|z|<1 / 2$, we have

$$
\Phi_{P}(z)=\eta(z)\left(Y(z)+\frac{q z \eta(z)}{1-q z \eta(z)} X(z)\right) .
$$

Proof. See [3].

Theorem 1 For all $z$ such that $|z|<1 / 2$, we have

$$
L(z)=\frac{\eta(z)}{1-q z \eta(z)} U X(z)
$$

and

$$
\Phi_{L(z)}(z)=I+\frac{z}{1-\rho q z \eta(z)} L(z)
$$


Proof. Let us fix $z$ such that $|z|<1 / 2$. Since $R W=S W=0$, we have by definition of $X(z)$ and $Y(z)$

$$
\begin{aligned}
& R Y(z)=R X(z) \\
& S Y(z)=S X(z),
\end{aligned}
$$

which gives, by definition of matrix $U$,

$$
U Y(z)=U X(z) .
$$

The definition of $L(z)$ and Lemma 4 lead to

$$
\begin{aligned}
L(z) & =U \Phi_{P}(z) \\
& =\eta(z)\left(U Y(z)+\frac{q z \eta(z)}{1-q z \eta(z)} U X(z)\right) \\
& =\eta(z)\left(1+\frac{q z \eta(z)}{1-q z \eta(z)}\right) U X(z) \\
& =\frac{\eta(z)}{1-q z \eta(z)} U X(z)
\end{aligned}
$$

which proves (8).

Consider now the successive powers $L^{k}(z)$ of matrix $L(z)$. Observing that

$$
\begin{aligned}
X(z) R X(z) & =X(z) \\
X(z) S X(z) & =p z \eta(z) X(z)
\end{aligned}
$$

we easily get

$$
\begin{aligned}
L^{2}(z) & =\left(\frac{\eta(z)}{1-q z \eta(z)}\right)^{2}(U X(z))^{2} \\
& =\left(\frac{r \eta(z)}{c(1-q z \eta(z))}\right)^{2}(p R X(z)-q S X(z))^{2} \\
& =\left(\frac{r \eta(z)}{c(1-q z \eta(z))}\right)^{2} p(1-q z \eta(z))(p R X(z)-q S X(z)) \\
& =\frac{r p \eta(z)}{c} L(z) \\
& =\rho q \eta(z) L(z) .
\end{aligned}
$$

$\mathrm{RR} \mathrm{n}^{\circ} 4281$ 
It follows by induction that for every $k \geq 0$,

$$
L^{k+1}(z)=(\rho q \eta(z))^{k} L(z) .
$$

Since $|z|<1 / 2$, it is easy to check from Relation (6) that $|\eta(z)| \leq 2$ and so that $|q z \eta(z)|<1$. Thus, we get

$$
\begin{aligned}
\Phi_{L(z)}(z) & =\sum_{k=0}^{\infty} L^{k}(z) z^{k} \\
& =I+z \sum_{k=0}^{\infty}(\rho q z \eta(z))^{k} L(z) \\
& =I+\frac{z}{1-\rho q z \eta(z)} L(z),
\end{aligned}
$$

which completes the proof.

Theorem 2 For $|z|<\min \{1 / 2, c /(q r+c)\}$, we have

$$
\Phi_{T}(z)=\eta(z)\left(Y(z)+\frac{q z \eta^{2}(z)\left((1+\rho-\rho q z \eta(z)) X(z)-\frac{r}{c} W X(z)\right)}{(1-q z \eta(z))(1-\rho q z \eta(z))}\right) .
$$

Proof. Let $z$ be such that $|z|<\min \{1 / 2, c /(q r+c)\}$. Replacing Relations (7) and (9) in Relation (5), we obtain

$$
\Phi_{T}(z)=\eta(z)\left(Y(z)+\frac{q z \eta(z)}{1-q z \eta(z)} X(z)\right)\left(I+\frac{z}{1-\rho q z \eta(z)} L(z)\right) .
$$

Now, since $V R=0$ and $V S=R$, we obtain from Relations (10) and (11)

$$
\begin{aligned}
& Y(z) R X(z)=X(z) \\
& Y(z) S X(z)=p z \eta(z) X(z)+W X(z) .
\end{aligned}
$$

We get from (8),

$$
\begin{aligned}
Y(z) L(z) & =\frac{r \eta(z)}{c(1-q z \eta(z))}(p Y(z) R X(z)-q Y(z) S X(z)) \\
& =\rho q \eta(z) X(z)-\frac{r q \eta(z)}{c(1-q z \eta(z))} W X(z)
\end{aligned}
$$


and using (10) and (11),

$$
\begin{aligned}
X(z) L(z) & =\frac{r \eta(z)}{c(1-q z \eta(z))}(p X(z) R X(z)-q X(z) S X(z)) \\
& =\rho q \eta(z) X(z) .
\end{aligned}
$$

Putting (14) and (15) in (13), we obtain

$$
\begin{aligned}
\Phi_{T}(z)= & \eta(z) Y(z)+\frac{z \eta(z)}{1-\rho q z \eta(z)} Y(z) L(z) \\
& +\frac{q z \eta^{2}(z)}{1-q z \eta(z)} X(z)+\frac{q z^{2} \eta^{2}(z)}{(1-q z \eta(z))(1-\rho q z \eta(z))} X(z) L(z) \\
= & \eta(z) Y(z)+\frac{\rho q z \eta^{2}(z)}{1-\rho q z \eta(z)} X(z)-\frac{r q z \eta^{2}(z)}{c(1-q z \eta(z))(1-\rho q z \eta(z))} W X(z) \\
& +\frac{q z \eta^{2}(z)}{1-q z \eta(z)} X(z)+\frac{\rho q^{2} z^{2} \eta^{3}(z)}{(1-q z \eta(z))(1-\rho q z \eta(z))} X(z) \\
= & \eta(z) Y(z)-\frac{r q z \eta^{2}(z)}{c(1-q z \eta(z))(1-\rho q z \eta(z))} W X(z) \\
& +\frac{q z \eta^{2}(z)(1+\rho-\rho q z \eta(z))}{(1-q z \eta(z))(1-\rho q z \eta(z))} X(z),
\end{aligned}
$$

which is the desired result.

\section{Explicit solution}

We obtain in this section a closed-form expression for $T_{0, j}^{n}$ and so for $F_{j}(x)$. For that purpose, we need the following well-known lemma which gives an analytical expression of the powers of $\eta(z)$.

Lemma 5 For every $k \geq 1$ and $|z| \leq 1 / 4$, we have

$$
C^{k}(z)=\sum_{n=0}^{\infty} s(k, n) z^{n}
$$

$\mathrm{RR} \mathrm{n}^{\circ} 4281$ 
where

$$
s(k, n)=k \frac{(2 n+k-1) !}{n !(n+k) !} .
$$

Proof. See [5], page 154 .

The integers $s(k, n)$ are referred to as the ballot numbers.

Theorem 3 For every $j \geq 0$,

$$
T_{0, j}^{n}= \begin{cases}0 & \text { if } n<j, \\ \frac{p^{j}}{(1-\rho) q^{j}} \sum_{k=0}^{\left\lfloor\frac{n-j}{2}\right\rfloor} s(n-2 k+1, k) p^{k} q^{n-k}\left(1-\rho^{n-j-2 k+1}\right) & \text { if } n \geq j,\end{cases}
$$

where $\lfloor u\rfloor$ denotes the largest integer less than or equal to the real number $u$.

Proof. Let $z$ be such that $|z|<\min \{1 / 2, c /(q r+c)\}$. Since the first row of the matrix $W X(z)$ has all its entries equal to zero, we have from Relation (12), for every $j \in \mathbb{N}$,

$$
\left(\Phi_{T}(z)\right)_{0, j}=\eta(z) Y_{0, j}(z)+\frac{q z \eta^{2}(z)(1+\rho-\rho q z \eta(z))}{(1-q z \eta(z))(1-\rho q z \eta(z))} X_{0, j}(z) .
$$

By definition of $X(z)$ and $Y(z)$ we easily check that

$$
X_{0, j}(z)=Y_{0, j}(z)=(p z \eta(z))^{j} .
$$

So, we obtain

$$
\left(\Phi_{T}(z)\right)_{0, j}=\frac{p^{j} z^{j} \eta^{j+1}(z)}{(1-q z \eta(z))(1-\rho q z \eta(z))} .
$$

For $|z|<1 / 2$, we have $|q z \eta(z)|<1$ and therefore, using the Cauchy product of two series, we obtain

$$
\begin{aligned}
\left(\Phi_{T}(z)\right)_{0, j} & =\frac{p^{j} z^{j} \eta^{j+1}(z)}{(1-q z \eta(z))(1-\rho q z \eta(z))} \\
& =p^{j} z^{j} \eta^{j+1}(z) \sum_{n=0}^{\infty} \sum_{k=0}^{n}(\rho q z \eta(z))^{k}(q z \eta(z))^{n-k}
\end{aligned}
$$




$$
\begin{aligned}
& =\frac{p^{j} z^{j} \eta^{j+1}(z)}{1-\rho} \sum_{n=0}^{\infty}(q z \eta(z))^{n}\left(1-\rho^{n+1}\right) \\
& =\frac{p^{j}}{(1-\rho) q^{j}} \sum_{n=j}^{\infty} q^{n} z^{n} \eta^{n+1}(z)\left(1-\rho^{n-j+1}\right) .
\end{aligned}
$$

From Lemma 5, we have

$$
\eta^{n+1}(z)=C^{n+1}\left(p q z^{2}\right)=\sum_{k=0}^{\infty} s(n+1, k) p^{k} q^{k} z^{2 k}
$$

which leads, by changing the order of summations, to

$$
\begin{aligned}
\left(\Phi_{T}(z)\right)_{0, j} & =\frac{p^{j}}{(1-\rho) q^{j}} \sum_{k=0}^{\infty} \sum_{n=j}^{\infty} s(n+1, k) p^{k} q^{n+k} z^{n+2 k}\left(1-\rho^{n-j+1}\right) \\
& =\frac{p^{j}}{(1-\rho) q^{j}} \sum_{k=0}^{\infty} \sum_{n=2 k+j}^{\infty} s(n-2 k+1, k) p^{k} q^{n-k} z^{n}\left(1-\rho^{n-2 k-j+1}\right)
\end{aligned}
$$

Exchanging again the order of summations, we get

$$
\left(\Phi_{T}(z)\right)_{0, j}=\frac{p^{j}}{(1-\rho) q^{j}} \sum_{n=j}^{\infty} z^{n} \sum_{k=0}^{\left\lfloor\frac{n-j}{2}\right\rfloor} s(n-2 k+1, k) p^{k} q^{n-k}\left(1-\rho^{n-2 k-j+1}\right),
$$

which completes the proof.

Using Relation (3) and Theorem 3, we obtain, for every $j \in \mathbb{N}$ and $x \geq 0$,

$$
F_{j}(x)=\frac{p^{j}}{q^{j}} \sum_{n=j}^{\infty} \exp (-\theta x) \frac{(\theta x)^{n}}{n !} \sum_{k=0}^{\left\lfloor\frac{n-j}{2}\right\rfloor} s(n-2 k+1, k) p^{k} q^{n-k}\left(1-\rho^{n-2 k-j+1}\right)
$$

that is,

$$
F_{j}(x)=\frac{p^{j}}{q^{j}} \sum_{n=j}^{\infty} \exp (-\theta x) \frac{(\theta x)^{n}}{n !} \sum_{k=0}^{\left\lfloor\frac{n-j}{2}\right\rfloor} \frac{n-2 k+1}{n-k+1}\left(\begin{array}{l}
n \\
k
\end{array}\right) p^{k} q^{n-k}\left(1-\rho^{n-2 k-j+1}\right) .
$$

$\mathrm{RR} \mathrm{n}^{\circ} 4281$ 
For $i, j \in \mathbb{N}$ and $t \geq 0$, we denote by $P_{i, j}(t, \lambda, \mu)$ the transition probability at time $t$ of the $\mathrm{M} / \mathrm{M} / 1$ queue with arrival rate $\lambda$ and service rate $\mu$, that is

$$
P_{i, j}(t, \lambda, \mu)=\operatorname{Pr}\left\{X_{t}=j \mid X_{0}=i\right\}
$$

It has been shown in [3] that

$$
P_{0, j}(t, \lambda, \mu)=\frac{p^{j}}{q^{j}} \sum_{n=j}^{\infty} \exp (-(\lambda+\mu) t) \frac{(\lambda+\mu)^{n} t^{n}}{n !} \sum_{k=0}^{\left\lfloor\frac{n-j}{2}\right\rfloor} s(n-2 k+1, k) p^{k} q^{n-k}
$$

and, in particular, for $j=0$,

$$
P_{0,0}(t, \lambda, \mu)=1-\frac{\lambda}{\mu} \sum_{n=1}^{\infty} \exp (-(\lambda+\mu) t) \frac{(\lambda+\mu)^{n} t^{n}}{n !} \sum_{k=0}^{\left\lfloor\frac{n-1}{2}\right\rfloor}\left(\begin{array}{c}
2 k \\
k
\end{array}\right) \frac{p^{k+1} q^{k}}{k+1} .
$$

In the following corollaries, we show that the distribution of the stationary buffer content of the fluid queue can be expressed as a function of the transient behavior of the $\mathrm{M} / \mathrm{M} / 1$ queue.

Corollary 1 For every $j \in \mathbb{N}$ and $x \geq 0$, we have

$$
F_{j}(x)=P_{0, j}\left(\frac{x}{r-c}, \lambda, \mu\right)-\rho^{j+1} \exp \left(-\left(\frac{\mu}{r}-\frac{\lambda}{c}\right) x\right) P_{0, j}\left(\frac{x}{r-c}, \frac{\mu c}{r}, \frac{\lambda r}{c}\right) .
$$

Proof. The proof is immediate from Relations (17) and (18).

Corollary 2 For every $x \geq 0$, we have

$$
\operatorname{Pr}\{Q \leq x\}=\frac{r F_{0}(x)}{r-c}-\frac{c(1-\rho)}{r-c} .
$$

Proof. Consider the differential system (1). By summing over index $j$, we get

$$
-c F_{0}^{\prime}(x)+(r-c) \sum_{j=1}^{\infty} F_{j}^{\prime}(x)=0
$$


Integrating from 0 to $x$, we obtain

$$
(r-c) \sum_{j=1}^{\infty}\left(F_{j}(x)-F_{j}(0)\right)=c\left(F_{0}(x)-F_{0}(0)\right) .
$$

Since $F_{j}(0)=0$ for $j \geq 1$ and $F_{0}(0)=1-\rho$, we have

$$
\operatorname{Pr}\{Q \leq x\}=\sum_{j=0}^{\infty} F_{j}(x)=\frac{1}{r-c}\left(r F_{0}(x)-c(1-\rho)\right) .
$$

The result follows by using Relation (17) for $j=0$.

\section{$5 \quad$ Finite buffer case}

We consider in this section the case where the capacity $B$ of the buffer is finite. We denote by $Q_{B}$ the stationary fluid buffer level and we define $F_{j, B}(x)=\operatorname{Pr}\left\{X=j, Q_{B} \leq x\right\}$. The following result gives an interesting relation between $F_{j, B}(x)$ and $F_{j}(x)$.

Theorem 4 If $\rho<1$, we have for every $j \in \mathbb{N}$ and $0 \leq x<B$,

$$
F_{j, B}(x)=(1-\lambda / \mu) \frac{F_{j}(x)}{F_{0}(B)} .
$$

Proof. See [6].

Using this result and Corollary 2, we easily get, if $\rho<1$ and $0 \leq x<B$,

$$
\operatorname{Pr}\left\{Q_{B} \leq x\right\}=\frac{(1-\lambda / \mu)\left(r F_{0}(x)-c(1-\rho)\right)}{(r-c) F_{0}(B)} .
$$

The overflow probability is thus given by

$$
\operatorname{Pr}\left\{Q_{B}=B\right\}=1-\operatorname{Pr}\left\{Q_{B}<B\right\}=\frac{c(1-\rho)}{r-c}\left(\frac{1-\lambda / \mu}{F_{0}(B)}-1\right) .
$$

$\mathrm{RR} \mathrm{n}^{\circ} 4281$ 


\section{References}

[1] Adan, I. and Resing, J. (1996). Simple analysis of a fluid queue driven by an M/M/1 queue. Queueing Systems 22, 171-174.

[2] Barbot, N. And Sericola, B. (2001). Transient analysis of a fluid queue driven by an M/M/1 queue. In Proc. 9th International Conference on Telecommunication Systems: Modeling and Analysis (ICTS'9), Dallas, TX, USA.

[3] Leguesdron, P., Pellaumail, J., Rubino, G. and Sericola, B. (1993). Transient analysis of the $\mathrm{M} / \mathrm{M} / 1$ queue. Adv. Appl. Prob. 25, 702-713.

[4] Mitra, D. (1988). Stochastic theory of a fluid model of producers and consumers coupled by a buffer. Adv. Appl. Prob. 20, 646-676.

[5] Riordan, J. (1968). Combinatorial identities, John Wiley, New York.

[6] Sericola, B. (2001). A finite buffer fluid queue driven by a Markovian queue. Queueing Systems 38, 213-220.

[7] Sericola, B. and Tuffin, B. (1999). A fluid queue driven by a Markovian queue. Queueing Systems 31, 253-264.

[8] Stern, T. E. And Elwalid, A. I. (1991). Analysis of separable Markov-modulated rate models for information-handling systems. Adv. Appl. Prob. 23, 105-139.

[9] Van Doorn, E. A. And Scheinhardt, W. R. W. (1997). A fluid queue driven by an infinite state birth-death process. In Proc. 15th International Teletraffic Congress (ITC'15), Washington, DC, USA, ed. V. Ramaswami and P. E. Wirth, Elsevier.

[10] Virtamo, J. and Norros, I. (1994). Fluid queue driven by an M/M/1 queue. Queueing Systems 16, 373-386. 
Unité de recherche INRIA Lorraine, Technopôle de Nancy-Brabois, Campus scientifique, 615 rue du Jardin Botanique, BP 101, 54600 VILLERS LÈS NANCY

Unité de recherche INRIA Rennes, Irisa, Campus universitaire de Beaulieu, 35042 RENNES Cedex

Unité de recherche INRIA Rhône-Alpes, 655, avenue de l'Europe, 38330 MONTBONNOT ST MARTIN

Unité de recherche INRIA Rocquencourt, Domaine de Voluceau, Rocquencourt, BP 105, 78153 LE CHESNAY Cedex

Unité de recherche INRIA Sophia-Antipolis, 2004 route des Lucioles, BP 93, 06902 SOPHIA-ANTIPOLIS Cedex

Éditeur

INRIA, Domaine de Voluceau, Rocquencourt, BP 105, 78153 LE CHESNAY Cedex (France)

http://www.inria.fr

ISSN 0249-6399 